2. Monobarium aluminate dissolves in barium hydroxide solutions with precipitation first of $7 \mathrm{BaO} .6 \mathrm{Al}_{2} \mathrm{O}_{3} .36 \mathrm{H}_{2} \mathrm{O}$, subsequently of $\mathrm{BaO} \cdot \mathrm{Al}_{2} \mathrm{O}_{3}$.$7 \mathrm{H}_{2} \mathrm{O}$ in the less basic and $\mathrm{BaO} \cdot \mathrm{Al}_{2} \mathrm{O}_{3} \cdot 4 \mathrm{H}_{2} \mathrm{O}$ in the more basic solutions.

3. Tribarium aluminate is rapidly hydrolyzed by water, with precipitation of $\mathrm{Ba}(\mathrm{OH})_{2} .8 \mathrm{H}_{2} \mathrm{O}, \mathrm{BaO}$.$\mathrm{Al}_{2} \mathrm{O}_{3} .7 \mathrm{H}_{2} \mathrm{O}$, and, subsequently, $2 \mathrm{BaO} . \mathrm{Al}_{2} \mathrm{O}_{3} .5 \mathrm{H}_{2} \mathrm{O}$.

4. All the hydrated barium aluminates dissolve in water and are hydrolyzed, with precipitation of hydrated alumina.

5. The hydrated barium aluminates dissolve in barium hydroxide solutions with eventual precipitation of the equilibrium solid phases, but frequently with preliminary separation of metastable intermediate solid phases.

6. The stable solid phases in the system $\mathrm{BaO}-\mathrm{Al}_{2} \mathrm{O}_{3}$ $\mathrm{H}_{2} \mathrm{O}$ at $30^{\circ} \mathrm{C}$ are: (a) gibbsite $\left(\mathrm{Al}_{2} \mathrm{O}_{3} \cdot 3 \mathrm{H}_{2} \mathrm{O}\right)$ over a range from approximately zero concentration to about $52 \mathrm{~g}$ of $\mathrm{BaO}$ and $2.8 \mathrm{~g}$ of $\mathrm{Al}_{2} \mathrm{O}_{3}$ per liter; (b) $\mathrm{Ba}(\mathrm{OH})_{2} .8 \mathrm{H}_{2} \mathrm{O}$ from $52.9 \mathrm{~g}$ of $\mathrm{BaO}$ and zero $\mathrm{Al}_{2} \mathrm{O}_{3}$ to about $55.5 \mathrm{~g}$ of $\mathrm{BaO}$ and $2.7 \mathrm{~g}$ of $\mathrm{Al}_{2} \mathrm{O}_{3}$ per liter; (c) probably $2 \mathrm{BaO} \cdot \mathrm{Al}_{2} \mathrm{O}_{3} .5 \mathrm{H}_{2} \mathrm{O}$ (but possibly $\mathrm{BaO}$.$\mathrm{Al}_{2} \mathrm{O}_{3} \cdot 4 \mathrm{H}_{2} \mathrm{O}$ or gibbsite) over the short range from $52 \mathrm{BaO}$ and $2.8 \mathrm{Al}_{2} \mathrm{O}_{3}$ to $55.5 \mathrm{BaO}$ and $2.7 \mathrm{Al}_{2} \mathrm{O}_{3}$.

7. $7 \mathrm{BaO} .6 \mathrm{Al}_{2} \mathrm{O}_{3} \cdot 36 \mathrm{H}_{2} \mathrm{O}$ is a metastable phase, not sufficiently stable to permit an accurate determination of its solubility.

8. $\mathrm{BaO} \cdot \mathrm{Al}_{2} \mathrm{O}_{3} \cdot 7 \mathrm{H}_{2} \mathrm{O}$ is also metastable, but it may exist in contact with solution for several months.

9. $\mathrm{BaO} \cdot \mathrm{Al}_{2} \mathrm{O}_{3} \cdot 4 \mathrm{H}_{2} \mathrm{O}$ is likewise metastable over the greater part, if not all, of its range, but its stability is greater than that of the higher hydrates.
10. $2 \mathrm{BaO} \cdot \mathrm{Al}_{2} \mathrm{O}_{3} .5 \mathrm{H}_{2} \mathrm{O}$ resembles $\mathrm{BaO} \cdot \mathrm{Al}_{2} \mathrm{O}_{3} \cdot 4 \mathrm{H}_{2} \mathrm{O}$ in its degree of stability in the metastable range.

11. No hydrate more basic than $2 \mathrm{BaO} \cdot \mathrm{Al}_{2} \mathrm{O}_{3} \cdot 5 \mathrm{H}_{2} \mathrm{O}$ was found.

\section{References}

[1] R. Stumper, Chimie \& industrie 22, 1067 to 83 (1929).

2] G. Grube and G. Heintz, Z. Electrochem. 41, 797 (1935).

[3] K. Akiyama, Z. Kajima, and H. Aiba, J. Soc. Chem. Ind. (Japan) 41, 218 (1938), and 42, 145 (1939); abstr. in Chem Abstr. 33, 325 and 7497 (1939).

[4] V. F. Zhuravlev, Tsement 1939, No. 8, 41; abstract in Chem. Abstr. 35, 595 (1941).

[5] F. L. Hunt and M. Temin, Radiology (Feb. 1927).

[6] G. W. Morey, U. S. Patent 1,688,054 (1928).

[7] H. V. Wartenburg and H. J. Reusch, Z. anorg. allgem. Chem. 20\%, 1 (1932).

[8] S. Wallmark and A. Westgren, Arkiv. Kemi, Mineral, Geol. 12B, No. 35 (1937).

[9] N. A. Toropov, Compt. rend. acad. sci. URSS 1935, 150.

[10] N. A. Toropov and M. M. Stukalova, Compt. rend. acad. sci. URSS, 24, 459 (1939).

[11] N. A. Toropov and M. M. Stukalova, Compt rend. acad. sci. URSS, 27,974 (1940).

[12] E. T. Carlson and L. S. Wells, J. Research NBS 41, 103 (1948) RP1908.

[13] E. Beckman, J. prakt. Chem. [2] 26, 385 and 474; 27, 126 (1883).

[14] G. Maekawa, J. Soc. Chem. Ind. (Japan) 44, 912 (1941); abstr. in Chem. Abstr. 42, 2536 (1948).

[15] G. Maekawa, J. Soc. Chem. Ind. (Japan) 45, 130 (1942).

[16] G. Malquori, Gazz. chim. ital. 56, 51 (1926).

[17] G. Gallo, Ind. ital. del cemento 17, 123 (1947).

[18] H. Le Chatelier, Experimental researches on the constitution of hydraulic mortars (1887) (Translated by J. L. Mack, 1905).

[19] A. Braniski, Rev. matériaux construction trav. publ. (Ed. C), No. 404, 154 (1949).

Washington, June 2, 1950.

\title{
Permeability of Glass Wool and Other Highly Porous Media
}

\section{By Arthur S. Iberall}

\begin{abstract}
An elementary treatment is developed for the permeability of fibrous materials of high porosities, based on the drag of the individual filaments. It is believed that the same treatment is valid for other highly porous media. A brief historical review is given of theories relating the permeability to the structure of porous media. The applicability of the currently accepted permeability theory, based on the hydraulic radius, only to media of low porosities is discussed. Both approaches may be extended to permit approximate correlation for intermediate porosities. For fibrous materials of high porosity, it is shown that the effect of fluid inertia results in a permeability that varies with flow even at low Reynolds number. The permeability to gaseous flow is also shown to vary with the absolute gas pressure. This variation is appreciable when the molecular mean free path is of the same order of magnitude as the separation between filaments or particles in the medium. Data suitable for the design of linear flowmeters utilizing fibrous materials of high porosity are given, including data on the useful porosity range of fibrous media.
\end{abstract}

\section{Introduction}

During the war there arose a need in the Bureau of Aeronautics, Department of the Navy, for rapid procurement of equipment suitable for field tests of diluter-demand oxygen regulators, which are used

1 This paper is a theoretical abstract of a report to the Bureau of Aeronautics, Navy Department [1]. Figures in brackets indicate the literature references at the end of this paper. by flight personnel at high altitudes. Due to difficulties in procurement, and certain disadvantages in the convenient use of commercially available flowmeters for the measurement of gaseous flow, the development of a suitable flowmeter was undertaken. After some preliminary consideration, efforts were centered on the development of a constant-resistance flowmeter utilizing a porous medium as the flow- 
resistance element. The major advantage of the constant-resistance (or constant-permeability) flowmeter is that its pressure drop is an indication of and proportional to the volume rate of flow, so that an instrument with linear response can be obtained.

Glass wool appeared to be a suitable porous medium because it is relatively inactive chemically, relatively nonhygroscopic, its fibers are strong and elastic, making in bulk a resilient mass that will retain its characteristics; and the fibers can be made in an almost unlimited range of sizes. A wide range of permeability is therefore obtainable that is absolutely essential for latitude in the design of flowmeters. In addition, the wool is inexpensive and easy to procure.

The development of these flowmeters included an investigation into the general flow characteristics of porous media, with particular reference to a medium consisting of randomly arrayed filaments. The primary concern in this paper is with the theoretical aspects of the permeability of highly porous media of this type.

\section{Permeability of Porous Media}

\section{Historical Review}

The characteristic law of fluid flow through a porous medium, for sufficiently low flows, is given by

$$
\frac{\Delta p}{L} \frac{A}{Q}=c \mu,
$$

where

$A=$ cross-sectional area of a cylindrical plug of a porous medium.

$L=$ length of the plug.

$\mathrm{Q}=$ volumetric rate of flow of fluid through the plug.

$\mu=$ absolute viscosity of the fluid.

$\Delta p=$ pressure drop between the ends of the plug.

$c=$ a constant (used indiscriminately throughout this paper). In eq 1 it is a factor characteristic of each particular medium. It is approximately a constant, increasing at high flows, and for gases, decreasing at low absolute pressures.

It is the elucidation of the principal dependence of the factor $c$ on the structure of the medium and its incidental dependence on the properties of the fluid that is the principal object in this paper. It will be useful to define the quantity $A \Delta p / L O$ as the resistivity of the medium for a given fluid, and its reciprocal $L Q / A \Delta p$ as the permeability. The quantity $c$ may then be referred to as the coefficient of resistivity of the porous medium, or its reciprocal $1 / c$ as the coefficient of permeability.

D'Arcy's experiments (in 1856) on the flow of water through sands led him to establish eq 1 , and it is commonly referred to as D'Arcy's equation.

Dupuit (in 1863) extended it to partially include dependence on the porosity of the medium. The porosity $\epsilon$ is defined as the ratio of the volume occu- pied by voids available to the fluid in a porous medium to the total volume. He argued that the apparent velocity $Q / A$ in a porous medium was less than the actual average velocity in the pore spaces, His expression was thus equivalent to

$$
\frac{\Delta p}{L} \frac{A}{Q}=c \frac{\mu}{\epsilon} .
$$

Slichter (in 1897) considered a granular bed as an equivalent system of capillary tubes. On this basis he derived the equation

$$
\frac{\Delta p}{L} \frac{A}{Q}=c \frac{\mu}{d^{2} F(\epsilon)},
$$

where

$d=$ dimension characteristic of the structure of the medium. In this instance, it is the diameter of the granule.

$F(\epsilon)=$ empirical function of the porosity.

Many investigators have subsequently attempted to obtain a generally applicable form of the porosity function.

The valuable methods of Stanton and Pannell (in 1914) of correlating data on flow through smooth circular pipes on the basis of the Reynolds number and other dimensionless groups, were followed by the concept of Schiller (in 1923) of a mean hydraulic radius that permitted correlating flow data on noncircular pipes. The mean hydraulic radius $r$ may be defined as the ratio of the volume of a medium filled with a fluid to the surface within the medium in contact with the fluid. Blake (in 1922) utilized the idea of a mean hydraulic radius in graphically correlating data on flow through granular beds in terms of a Reynolds number and other dimensionless groups. The results of Blake's work and the theoretical exposition of Kozeny (in 1927) on granular beds may be summarized in the formula

$$
\frac{\Delta p}{L} \frac{A}{Q}=c \frac{\mu s^{2}}{\epsilon^{3}},
$$

in which $s$ is the surface per unit volume of a porous medium in contact with the fluid $(s=\epsilon / r)$.

The Kozeny, or hydraulic radius, theory (eq 4) is intended to have general applicability to all porous media, because the only properties of the medium that remain buried in the constant $c$ involve the detailed structure of the medium, such as factors that take into account the shape and configuration of the fluid path or the shape and orientation of the material particles. In fact, experiments where this theory is definitely applicable show only moderate variation of this constant for a variety of shapes.

An extensive list of references, complete up to 1938, and more complete exposition of the problem can be found in reference [2].

In 1938 and 1939 [3], eq 4 was modified by Carman to the form

$$
\frac{\Delta p}{L} \frac{A}{Q}=c \frac{\mu s_{o}^{2}(1-\epsilon)^{2}}{\epsilon^{3}},
$$


in which $s_{o}$ is the "specific" surface exposed to the fluid (surface exposed to the fluid per unit volume of solid material).

The value of $c$ in eq 5 was found to be about 5 for granular materials of low porosities.

Equation 5 was applied to fibrous materials by Wiggins, Campbell, and Maass [4], and by Fowler and Hertel [5]. A clear exposition of the hydraulic radius theory leading to eq 5 may be also found in reference [4].

The latest modification of the hydraulic radius theory [6] involves the introduction of an orientation factor $\mathrm{O}$, which is defined as the averaged value of the square of the sine of the angle between a normal to the particle, fiber, or wall forming the microscopic flow channel and the macroscopic direction of flow. The resulting formula is

$$
\frac{\Delta p}{L} \frac{A}{Q}=\frac{\mu K_{o} s_{o}^{2}}{O} \frac{(1-\epsilon)^{2}}{\epsilon^{3}} .
$$

$K_{0}$ is a shape factor that should be the same for all channels of the same geometric shape and that should not vary markedly from shape to shape.

The Kozeny, or hydraulic radius, theory (from eq 4 onward) has thus been carried to a high degree of refinement. Many investigators, however, have not felt justified in using any relationship more complicated than the D'Arcy formula (eq 1), in which an empirical constant $1 / c$ (the coefficient of permeability) is obtained experimentally for each medium investigated.

A theory developed from a different point of view - which may be referred to as the drag theoryhas existed for some time without attracting much recognition or support. Emersleben (in 1925) attempted a mathematical solution of the hydrodynamic problem of the viscous drag of a fluid on a special array of parallel fibers. Burke and Plummer (in 1928) used the drag on spheres to obtain a law for the dependence of permeability of a porous medium on its porosity. The drag theory leads to a different expression for the dependence of resistivity on porosity than that given by the hydraulic radius theory. As existing data at that time and later, particularly on flow through sands, appeared to be consistent with the hydraulic radius theory, little attention has been given to the drag theory.

Actually, it appears that neither the hydraulic radius theory nor the drag theory can be entirely correct, but that each will bave a range of application. This concept is implicit in Emersleben's solution. Indeed, data on fibrous materials obtained by Sullivan in 1942 indicated that the hydraulic radius theory broke down at high porosities, where one may expect an elementary viscous drag theory to apply most satisfactorily.

It can be easily understood why there is a separate range of applicability of these two theories by considering the extreme cases. For an array of fibers or particles of large separation, the resistance to flow can be computed as the sum of the fluid drags on each element. As the elements are brought closer together, adjacent elements will modify the flow pattern surrounding a particular element. In principle, if account could be taken of the effect of the mutual interference between particles, the drag theory should be applicable to all porosities. However, in practice, this is beset by mathematical difficulties. Thus an elementary drag theory should fail when applied to low porosities.

In the hydraulic radius theory, the estimate of the resistance offered by a connected network of flow channels is based on the product of the area of solid surface in contact with fluid and an "equivalent" shearing stress acting on this area. The equivalent shearing stress is assumed to be that obtained from the normal velocity gradient that would exist at the walls of an equivalent channel formed between two parallel plates [4]. When the separation between the drag surfaces is small (more exactly, when the separation is small compared to the radius of curvature of a surface), a valid estimate is obtained. However, as the separation increases (higher porosities), the velocity gradient normal to the surface depends more and more on the ratio of the radius of curvature at the surface to the separation rather than on the distance to an adjacent wall. This is readily seen in the case of two concentric tubes with axial flow between them. With small separation, the hydraulic radius theory gives a true account of the flow resistance in the annulus. As the inner tube is shrunk, this is no longer true. Thus it turns out that the hydraulic radius theory is best applicable at low porosities and the drag theory at high porosities.

In the present investigation, expressions for the resistivity of fibrous materials have been derived from elementary consideration of the viscous drag of individual elements and were found to be moderately successful experimentally. This might be expected, as, in general, fibrous materials under moderate packing will still have rather high porosity. It is thus proposed that the same method of attack is suitable for all materials of high porosity.

The problem of intermediate porosities will be left untouched. It is possible, by semiempirical methods, to find expressions that may be expected to fit approximately the entire range of porosities, or, by perturbation methods, to extend the range of applicability of each theory individually, or finally, it may be possible by great effort, to find one single mathematical solution that is applicable at all porosities.

\section{Drag Theory of Permeability}

We will undertake to account for the permeability of a random distribution of circular cylindrical fibers of the same diameter on the basis of the drag on individual elements.

It will be assumed that the flow resistivity of all random distributions of the same fibers per unit volume will not differ, and that it will be the same as that obtained with an equipartition of fibers in three perpendicular directions, one of which is alon 
the direction of macroscopic flow. It will be further assumed that the separation between fibers, and the length of individual fibers are both large compared to the fiber diameter (high porosities), and that the disturbance due to adjacent fibers on the flow around any particular fiber is negligible.

If it be assumed that fluid inertial forces are negligible (low local Reynolds number) an equation can be derived by equating the pressure at two planes perpendicular to the direction of macroscopic flow to the viscous drag force on all elements between the planes. It is assumed that the pressure drop necessary to overcome the viscous drag is linearly additive for the various fibers, whether parallel or perpendicular to the flow.

It was estimated from Emersleben's paper [7] that the drag force per unit length of a single fiber surrounded by similar fibers all oriented along the direction of flow and with moderate separations is approximately given by

$$
f=4 \pi \mu v,
$$

where

$f=$ drag force per unit length of fiber.

$v=$ velocity of the fluid stream distant from the filament.

If it be assumed that there are $n$ filaments per unit volume, and that $n / 3$ filaments are arrayed in eacb of the three perpendicular directions, the total drag force in a unit volume due to the $n / 3$ filaments parallel to the flow can be equated to the pressure drop per unit length, so that

$$
\frac{\Delta p}{L}=\frac{4 \pi n}{3} \mu v .
$$

It is estimated from Oseen's solution for a cylinder perpendicular to a stream that partially takes into account fluid inertia (see Lamb's "Hydrodynamics") that the drag force for such filaments is given by

$$
f=\frac{4 \pi}{2-\ln R} \mu v
$$

in which

$\ln R=$ natural logarithm of the local Reynolds number.

$R=$ Reynolds number, defined as $d v \rho / \mu$.

$d=$ fiber diameter.

$\rho=$ fluid density.

The drag force on each of the two sets of $n / 3$ filaments per unit volume, arrayed perpendicular to the fluid flow, can be equated to the pressure drop per unit length, giving

$$
\frac{\Delta p}{L}=\frac{4 \pi n}{3(2-\ln R)} \mu v .
$$

Linear superposition or simple addition of the three sets of pressure drops necessary to overcome the drag of the three sets of filaments results in the total pressure gradient of

$$
\frac{\Delta p}{L}=\frac{4 \pi n}{3} \frac{4-\ln R}{2-\ln R} \mu v .
$$

The number of fibers $n$ per unit volume, which is also equal to the total fiber length per unit volume, may be eliminated, as the apparent density of a fibrous pack in vacuum $\sigma_{p}$ is equal to the product of the fiber volume and the fiber density $\sigma_{f}$, or

$$
\sigma_{p}=\frac{\pi}{4} d^{2} n \sigma_{f} .
$$

Eliminating $n$, eq 11 reduces to

$$
\frac{\Delta p}{L}=\frac{16 \mu v}{3} \frac{\sigma_{p}}{\sigma_{f} d^{2}} \frac{4-\ln R}{2-\ln R} .
$$

The velocity profile between fibers is assumed to be sufficiently flat for high porosities so that the velocity may be taken as constant. The velocity $v$ is therefore related to the volumetric rate of flow $Q$ and the macroscopic cross sectional area of a plug $A$ by the relation

$$
v=\frac{Q}{\epsilon A}
$$

It follows from the definition of the porosity $\epsilon$ that

$$
1-\epsilon=\frac{\sigma_{p}}{\sigma_{f}} .
$$

With the use of these two relations, eq 13 may be put in the form

$$
\begin{aligned}
\frac{\Delta p}{L} \frac{A}{Q} & =\frac{16 \mu}{3} \frac{1-\epsilon}{\epsilon d^{2}} \frac{4-\ln R}{2-\ln R}, \\
& =\frac{16 \mu}{3} \frac{\sigma_{p}}{\left(\sigma_{f}-\sigma_{p}\right) d^{2}} \frac{4-\ln R}{2-\ln R} .
\end{aligned}
$$

Although the derivation, as given, assumed an incompressible fluid, it can be readily shown that the derived equations are unchanged for a compressible fluid, flowing isothermally, if the volumetric flow at the arithmetic mean pressure $m$ is used in eq 16. It is therefore applicable to both liquids and gases. However, it will be shown later, that an additional correction must be made to eq 16 for gases at low absolute pressures.

If eq 6 is compared with eq 16 and their ratio is interpreted in terms of the shape factor of the hydraulic radius theory, it is possible to predict the general variation of the shape factor $K_{o}$ at high porosity. At low porosities, $K_{0}$ is constant, but at high porosities it should become asymptotic to

$$
K_{o}=\frac{c}{1-\epsilon}
$$

where $c$ is a constant. 
This prediction is qualitatively compatible with the data given in reference [6].

Another significant implication in eq 16 is that the resistivity (or permeability) is not a constant but varies slowly with Reynolds number. The theoretical variation of the resistivity is shown in table 1.

TABLE 1. Theoretical variation of resistivity with Reynolds number for a porous plug of randomly arrayed fibers

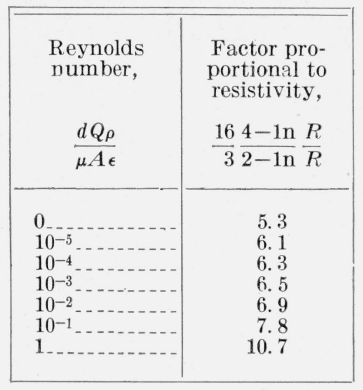

This slow variation of resistivity with flow is quite characteristic of many elements in which the flow is nominally viscous and was immediately found experimentally in the first glass wool flowmeters that were studied. While in general it is reasonable to assume that this effect is associated with fluid inertia, it is often difficult to account for it precisely. In fact, a common method of separating the resistance into two additive components, one proportional to the viscous resistance, and the other proportional to a kinetic energy loss, often fails in securing added precision in describing experimental results with linear flow elements.

In eq 16 , it is noteworthy that the effect has been associated with an intrinsic variation of drag with Reynolds number. An extremely interesting mathematical interpretation of eq 16 is possible. The slow variation of a logarithmic expression, as that give in eq 16, can be closely approximated by an exponential form.

$$
\frac{\Delta p}{Q}=c Q^{x}
$$

This type of relation is often successfully used for linear flow elements without apparent theoretical justification. At least in the case of highly porous fibrous media it has been shown to have theoretical validity.

The quantity $Q^{x}$ represents to a high degree the point variation of Reynolds number, where the best value of $x$ depends on the Reynolds number.

For the resistance law given by eq 16, the best value of $x$ is

$$
x=\frac{2}{(2-\ln R)(4-\ln R)} .
$$

In reference [1], eq 16 was used for comparison with experimental data on glass wool and fair agreement found, so that its essential validity appears estab- lished. While it is apparent that eq 16 was derived from elementary considerations, to which many refinements are possible, particularly as to the statistical distribution function for drag and secondorder effects of porosity, lack of extensive experimental data make these refinements dubious. As an illustration of the possible accuracy of eq 16 , some selected data of fair accuracy obtained subsequent to the issue of reference [1] permitted close fitting by the formula

$$
\frac{\Delta p}{L} \frac{A}{Q}=9.4 \mu \frac{\sigma_{p}}{\left(\sigma_{f}-\sigma_{p}\right) d^{2}} \frac{2.4-\ln R}{2-\ln R} .
$$

Although at first sight, the change in numerical constants appears drastic, it can be shown to represent moderately reasonable changes in the amount and relative weighting of the parallel and perpendicular fiber drags.

The main conclusion from all the experimental data obtained is that the coefficient of the resistivity function always fell in the approximate range of 5 to 10 , and that the variation of resistivity with Reynolds number is less than given by eq 16 , but not as small as given by eq 20 .

\section{Limits of Linearity}

It is well known that a laminar flow regime does not persist around elements producing drag in a fluid stream at Reynolds numbers much greater than 1 . Even at Reynolds numbers less than 1, the drag force will not remain strictly proportional to the velocity. Furthermore, even when drag is almost proportional to velocity for an indivdual element, this may not necessarily hold true for an array of similar elements, even though widely spaced.

The value of the resistivity given by eq 16 may therefore not be expected to apply for Reynolds numbers much greater than 1 . However, experimental data indicated no inconsistencies up to a value of Reynolds number even as great as 1.2. Therefore, as a round measure of the limit of flow linearity, a Reynolds number of 1 may be assumed.

The resistance of a given porous plug used as a flowmeter may therefore be expressed as

$$
\frac{\Delta p}{Q}=\left(\frac{\Delta p}{Q}\right)_{\max }\left(\frac{4-\ln R_{\max }-\ln Q / Q_{\max }}{2-\ln R_{\max }-\ln Q / Q_{\max }}\right)\left(\frac{2-\ln R_{\max }}{4-\ln R_{\max }}\right),
$$

where the subscript max refers to the values of quantities at the maximum or full scale flow.

\section{Pressure Variation of Resistivity at Low Pressure for Gases}

At low absolute pressures, the flow of gas along a tube does not obey Poiseuille's law, the flow being somewhat larger for a given pressure drop than predicted by this law. At low gas pressures the resistance to flow in a tube may be derived by the methods of statistical mechanies [8]. 
The essential idea is that at low pressures, where the separation between walls is of the order of the mean free path, molecules will suffer few collisions with each other, and therefore should be capable of being urged to drift, or "flow", by an infinitesimal pressure gradient. However, there will be a loss of momentum suffered by molecules that collide with a stationary boundary (the tube wall). This loss of momentum is computed by assuming that, with a finite pressure gradient, the velocity of a molecule consists of its drift, or flow, velocity, superimposed on its Maxwellian thermal velocity; and that as a result of collisions with a wall, the molecule will be diffusely reflected, that is, it will give up its drift velocity and be reflected with some random Maxwellian velocity. The average velocity profile for gas flowing at low absolute pressures in a tube will therefore consist of a flat portion between the walls of constant velocity equal to the drift velocity, and a portion near the walls that falls very rapidly to zero, so that the velocity profile looks essentially flat-topped. This represents the model for molecular "drift" flow in a tube. At high pressures, viscosity is used to account for the flow resistance.

Knudsen found that, for a given pressure gradient at any absolute pressure, a large fraction of the drift velocity can be added to the "viscous" velocity to obtain a composite velocity in good agreement with experiment. The fraction of the computed drift velocity was semiempirically determined to range from 0.81 to 1 , depending on the ratio of mean free path to wall separation. The effect of this superposition leads to a result that encompasses the entire pressure range. One term, the Knudsen term, becomes predominant at low pressures, while the other term, the Poiseuille term, becomes predominant at high pressures. In the intermediate pressure range (above $1 \mathrm{~mm} \mathrm{Hg}$ absolute pressure), although small, the Knudsen term is not negligible.

Application of the same theory to porous media of a random filament nature leads to the result that the molecular drift flow at low pressure $Q_{m}^{\prime}$ is approximately given by

$Q_{m}^{\prime}=\left(\frac{8}{9 \pi}\right)^{1 / 2} \frac{A \Delta p}{L} \frac{d\left(1-\sigma_{p} / \sigma_{f}\right)^{2} \sigma_{f}}{\sigma_{p}}\left(\frac{p}{\rho}\right)_{o}^{1 / 2}\left(\frac{1}{p_{o}}\right) \frac{p_{o}}{p_{m}}$,

where subscript $m$ refers to conditions at the mean pressure, and

$p_{0}=$ arbitrary reference pressure, chosen as 1 atmosphere.

$(p / \rho)_{0}=$ pressure to density ratio for the gas at the mean plug temperature.

Following Knudsen, 0.81 of the drift flow is simply added to the viscous flow of eq 16 , so that the total flow at mean conditions is

$$
\begin{aligned}
Q_{m}= & \frac{1}{5.3} \frac{2-\ln R}{4-\ln R} \frac{\left(\sigma_{f}-\sigma_{p}\right) d^{2}}{\sigma_{p}} \frac{A \Delta p}{\mu_{m} L} \\
& {\left[1+(.81)(10)\left(\frac{8}{9 \pi}\right)^{1 / 2} \frac{\sigma_{f}-\sigma_{p}}{\sigma_{f} d}\left(\frac{p}{\rho}\right)_{m}^{1 / 2} \frac{\mu_{m}}{p_{o}} \frac{p_{o}}{p_{m}}\right] . }
\end{aligned}
$$

The approximate value 10 was chosen for the resistivity function in the Knudsen term in the bracket.

The form of eq 23 is thus

$$
Q_{m}=c\left(1+b \frac{p_{o}}{p_{m}}\right) \Delta p,
$$

where $b$ is the coefficient of the Knudsen term.

Experimentally, a linear variation of permeability $Q_{m} / \Delta p$ with the reciprocal mean pressure ratio $p_{o} / p_{m}$ was always obtained. In fact, fair agreement was found with the theoretically computed cofficient $b$ in practically all cases, although this result may have been purely fortuitous.

\section{Range of Useful Porosities of Fibrous Materials}

The following section, largely based on speculative considerations, is irrelevant to the development of the theory of permeability of porous media. However, it is of utility in the design of flowmeters. In a flowmeter, it is desirable to pack the fibrous material to such a density that it will remain relatively rigid under vibration, shock, or differential pressure overload.

The following theoretical and experimental conclusions, while tentative, furnish a rough guide in determining usable porosity limits for design purposes :

1. The upper limit to the porosity of a fibrous material is obtained with a free pack and is approximately 0.98 . The minimum ratio of free pack to fiber density $\sigma_{p} / \sigma_{f}$ is thus approximately 0.02 . The usable upper limit of porosity is set by other design considerations.

2. The lower limit to the porosity of a fibrous material is approximately 0.50 .

3 . To a crude approximation, the compressibility of all fibrous materials is the same, and equals $0.0021 \mathrm{lb} / \mathrm{in}^{3}$ of pack per pounds per square inch of pressure load.

4. Fibrous materials have fairly reproducible and constant compressibility characteristics on increasing load but show erratic hysteresis with decreasing load. Therefore all packing adjustments on fibrous plugs should be made with increasing load.

The approximate constancy of the free-pack density, it is believed, is related to the geometric-mechanical problem of the minimum number of filaments that must be introduced into a given volume to bind and form a stable pack.

The lower limit to the porosity of fibrous materials may be estimated by considering a close packed array of fibers perpendicular to each other in three directions. The porosity for such an array was computed to be 0.41 . Experimentally, porosities as low as 0.5 were obtained. While lower porosities can be obtained, it is believed that serious compression of the material rather than fiber bending would take place.

The approximate constancy of compressibility of various materials is probably related to the similar small variation in the ratio of elastic modulus to 
density for many elastic materials. Various models for the compressibility of a fibrous pack demonstrate the dependence of pack compressibility on this parameter.

In lieu of any other information, the variation of pack density with pressure loading for increasing load may be taken as

where

$$
\sigma_{p}=0.02 \sigma_{f}+0.0021 P
$$

$\sigma_{p}=$ pack density.

$\sigma_{f}=$ density of the fiber (in the same units).

$P=$ pressure loading. The constant 0.0021 has the units lb/in. ${ }^{3} /$ psi.

The theoretical range of pack density ratio is approximately 0.02 to 0.50 . The practical range of pack density ratio for flowmeter design is probably 0.1 to 0.3 .

\section{Design of Linear Flowmeters}

A convenient procedure is outlined in this section for designing a linear flowmeter on the basis of the theory developed in section II. It is assumed that the basic problem is to design a linear flowmeter that will possess a desired resistance $\Delta p / Q$, which is constant within given limits over a desired flow range $O-Q_{\max }$ for a given fluid. To meet these requirements, compromise choices must be made of fiber diameter, cross-sectional area of the flowmetering plug, plug density, and plug length.

A scheme, involving the use of a simple chart, has been evolved for design purposes. In the interests of clarity, the theory by which the design chart was devised will be sketched.

Equation 23 sets forth the law of isothermal gas flow for a fibrous plug as

$$
\begin{aligned}
Q_{m}= & \frac{1}{5.3} \frac{2-\ln R}{4-\ln R} \frac{\left(\sigma_{f}-\sigma_{p}\right) d^{2}}{\sigma_{p}} \frac{A \Delta p}{\mu_{m} L}[1+ \\
& \left.(.81)(10)\left(\frac{8}{9 \pi}\right)^{1 / 2} \frac{\sigma_{f}-\sigma_{p}}{\sigma_{f} d}\left(\frac{p}{\rho}\right)_{m}^{1 / 2} \frac{\mu_{m}}{p_{o}} \frac{p_{o}}{p_{m}}\right]
\end{aligned}
$$

The same equation holds for liquids, except that the bracketed expression bas the value unity.

As it is assumed that this law holds only for high porosities, the small correction term in the bracket may be evaluated for a porosity of unity so that eq 23 , for gases, becomes

$$
\begin{aligned}
Q_{m}= & \frac{1}{5.3} \frac{2-\ln R}{4-\ln R} \frac{\sigma_{f}-\sigma_{p}}{\sigma_{p}} d^{2} \frac{A}{\mu_{m} L} \\
& {\left[1+\frac{4.3}{d}\left(\frac{p}{\rho}\right)_{m}^{1 / 2} \frac{\mu_{m}}{p_{o}} \frac{p_{o}}{p_{m}}\right] \Delta p . }
\end{aligned}
$$

It will be convenient, in this section, to drop the subscript $m$, which denotes mean isothermal conditions, leaving it understood.

From the definition, the Reynolds number is

$$
R=\frac{d \rho Q \sigma_{f}}{\mu A\left(\sigma_{f}-\sigma_{p}\right)} .
$$

These equations may be regrouped by the following definitions:

where

$$
L_{o}=L \frac{\sigma_{p}}{\sigma_{f}-\sigma_{p}}=L \frac{1-\epsilon}{\epsilon},
$$

$L=$ actual plug length.

$L_{o}=$ fictitious plug length (length of plug if compressed to a porosity of 0.50 ).

$$
M_{o}=\frac{M_{\max }}{R_{\max }} \frac{\sigma_{f}}{\sigma_{f}-\sigma_{p}}=\frac{M_{\max }}{R_{\max }} \frac{1}{\epsilon},
$$

in which

$$
\begin{aligned}
& M=\text { mass flow }(M=\rho Q) . \\
& \text { flowmeter is to be used. } \\
& \left.\begin{array}{rl}
\left(\frac{Q}{\Delta p} L_{o}\right)_{o} & =\frac{1}{5.3} \frac{(\mu A)^{3}}{\left(\mu M_{o}\right)^{2}} \\
& =\frac{1}{5.3}\left(\frac{d}{\mu}\right)^{3} \mu M_{o}
\end{array}\right\}
\end{aligned}
$$$$
M_{\max }=\text { maximum mass flow for which the }
$$$$
R_{\max }=\text { corresponding maximum Reynolds num- }
$$

$M_{0}=$ fictitious flow (mass flow at which the Reynolds number is unity if the medi-

The quantity $\left(Q L_{o} / \Delta p\right)_{o}$ is a fictitious value for the flow per unit pressure gradient (the value for a Reynolds number of zero). It is closely related to the true value by the relation

$$
\frac{Q L_{o}}{\Delta p}=\frac{2-\ln R}{4-\ln R}\left(\frac{Q}{\Delta p} L_{o}\right)_{o}\left[1+\left(\frac{4.3}{p_{o}}\right)\left(\frac{p}{\rho}\right)^{1 / 2}\left(\frac{\mu}{d}\right) \frac{p_{o}}{p}\right] .
$$

The definitions, eq 27 through 30 , and eq 31 contain the desired solution.

Equation 30 may be plotted as a generalized chart applicable to all fluids, or, by choice of fluid parameters, as a design chart suitable for a specific fluid. It is presented in generalized form in figure 1 as a logarithmic plot of $(Q / \Delta p) L_{o}$ against $\mu M_{0}$ for constant values of $\mu A$ and $d / \mu$ respectively. The use of figure 1 or eq 30 leads to values of $A, L$, and $d$ for a porous plug that nominally will have the desired resistance $\Delta p / Q$. The best choice of these parameters depends upon the deviations from constant resistance that may be tolerated.

As the permeability theory developed has only nominal certainty, it is not to be expected that a desired resistance will be experimentally obtained with any great accuracy, so that in general, a moderate amount of resistance adjustment will be found necessary. (Illustratively, this may be accomplished by variation of the weight of glass wool used.) Therefore little distinction need be made as to the nominal design value of $\Delta p / Q$. The value $(\Delta p / Q)$ 。 may thus be regarded as the resistance at full scale, 


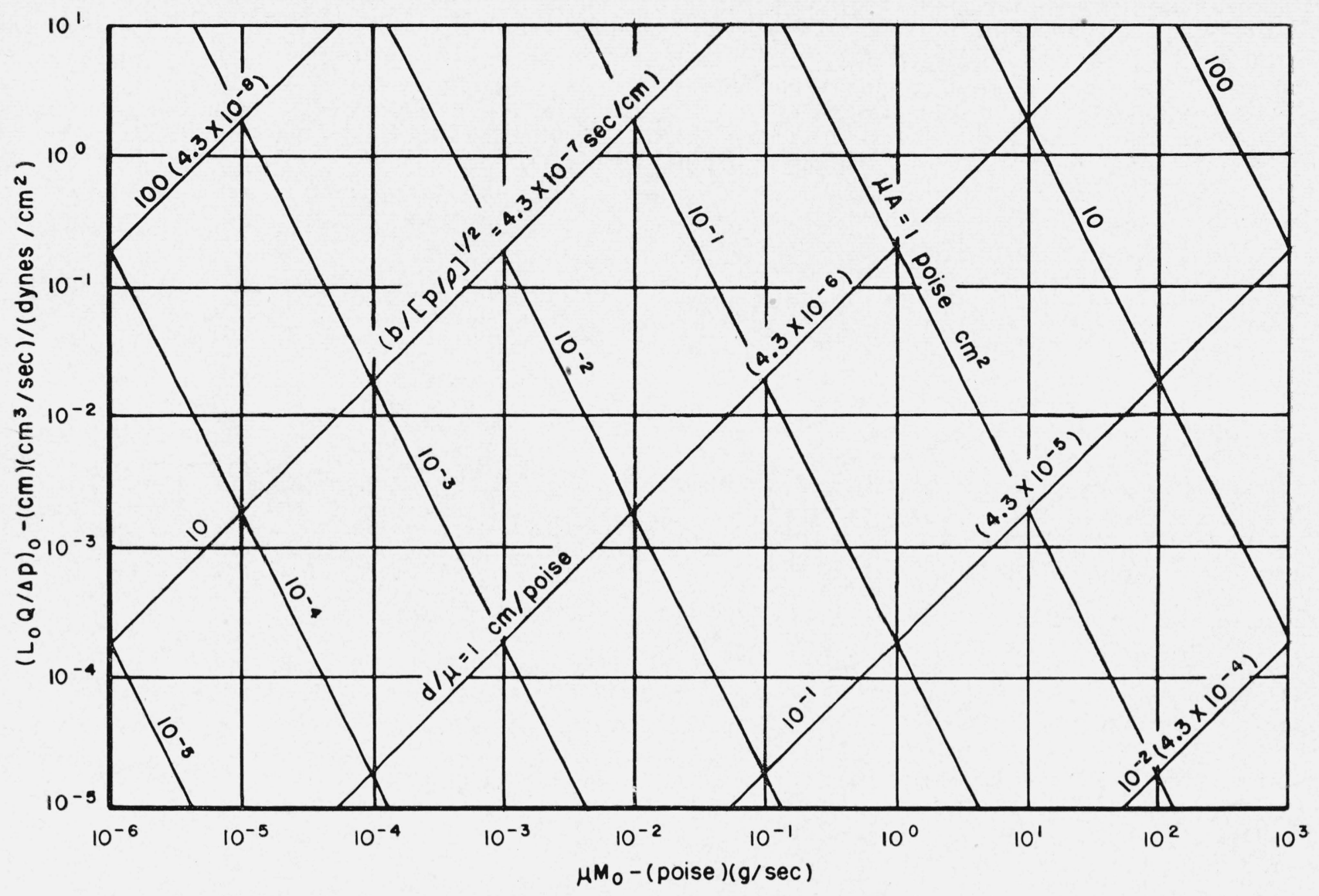

Figure 1. Design chart for lineur flowmeters

(See text for explanation of its use.)

establishing a nominal straight line calibration for the flowmeter. The variation of $(2-\ln R) /(4-\ln R)$ with flow may be regarded as determining the expected deviation from linearity, which may be characterized by its maximum value as either a quadratic deviation, or, more accurately, as an exponential deviation. The maximum deviation from a straight line fitted to the maximum flow and pressure drop is given in table 2, computed on the basis of eq 16. Any desired degree of linearity therefore determines a maximum value of Reynolds number to use for design purposes.

TABLE 2. Theoretical maximum deviation from linearity for a fibrous plug flowmeter

\begin{tabular}{|c|c|c|}
\hline $\begin{array}{l}\text { Reynoldsnum- } \\
\text { ber at full scale } \\
\text { flow, } R_{\max }\end{array}$ & $\begin{array}{l}\text { Maximum dif- } \\
\text { ference between } \\
\text { true flow and } \\
\text { linear approxi- } \\
\text { mation }\end{array}$ & $\begin{array}{l}\text { Location of } \\
\text { maximum }\end{array}$ \\
\hline $\begin{array}{l}1 \\
10^{-1} \\
10^{-2} \\
10^{-3} \\
10^{-4} \\
10^{-5} \\
10^{-6}\end{array}$ & $\begin{array}{c}\text { Percentage of } \\
\text { full scale flow } \\
6.5 \\
2.2 \\
1.1 \\
0.7 \\
.5 \\
.3 \\
.3\end{array}$ & $\begin{array}{c}\text { Percentage of } \\
\text { full scale flow } \\
48 \\
43 \\
41 \\
40 \\
40 \\
40 \\
39\end{array}$ \\
\hline
\end{tabular}

For gases, eq 31 indicates a change in resistance with absolute pressure, which depends on the fiber diameter $d$. Any desired degree of uniformity in the resistance over a range of absolute pressures can be secured by proper selection of $d$.

Finally, practical consideration of size and the usable porosity range of 0.7 to 0.9 determine the length $L$ and cross-sectional area $A$.

For purposes of clarity, a design procedure is given in some detail below:

1. Determine the following constants of the fluid to be metered: $\mu, \rho, p / \rho$ (for gases).

2. Decide upon the maximum flow $Q_{\max }$ to be metered, the desired resistance $\Delta p / Q$, the maximum amount of nonlinear deviation that may be tolerated, and in the case of gases, the absolute pressure range in which the flowmeter is to be used, and the corresponding range of resistance $\Delta p / Q$ that may be tolerated.

3. Decide upon the maximum practical range that may be used for $A, L$, and $d$.

4. From table 2, choose the maximum value of $R_{\max }$ that will give the desired linearity. Smaller values of $R_{\max }$ will give better linearity but may require such large plug areas as to be impractical. A rough empirical criterion is to consider a design band 1 decade wide. Compute minimum and maxi- 
mum values of $M_{o}$ (eq 29) assuming minimum and maximum values for both $R_{\max }$ and $\epsilon$. The range of $\epsilon$ may be taken as 0.7 to 0.9 . Compute the corresponding values for $\mu M_{o}$ and mark out the vertical band corresponding to these limits in figure 1.

5 . For the maximum and minimum limits assumed for both $L$ and $\epsilon$, compute the corresponding limits for $L_{o}$ (eq 28). Compute the corresponding values of $Q L_{o} / \Delta p$ and mark out the horizontal band corresponding to these limits in figure 1. Steps (4) and (5) result in a design rectangle on figure 1 within which a solution is possible.

6. Further limit this design rectangle by excluding regions of figure 1 representing greater and lesser area $A$ (really $\mu A$ ) than desired.

7. For gas flow, compute the maximum tolerable value of the coefficient of the Knudsen term $b$ and the corresponding minimum value of fiber diameter d. Exclude regions of figure 1 representing smaller values of $d$ (really $d / \mu)$. One may then choose design parameters corresponding to any point in the design region that has not been excluded.

8 . When the flowmeter is built and tested, adjustment of the resistivity can then be made by the principal technique of changing the weight of glass wool used.
Financial support for this investigation was provided by the Office of Naval Research under a project on Basic Instrumentation of Scientific Research. Grateful acknowledgement is also due W. A. Wildhack, at whose suggestion and under whose supervision the development of the glass wool flowmeter was carried on.

\section{References}

[1] National Bureau of Standards Report to the Bureau of Aeronautics, Navy Department, Washington, D. C., entitled, Linear pressure drop flowmeters for oxygen regulator test stands, reference 6.2/6211-2885 (Sept. 25, 1947).

[2] P. C. Carman, Trans. Institution Chem. Engrs. 15, 151 (1937); 16, 168 (1938).

[3] P. C. Carman, J. Soc. Chem. Ind. 5y, 225 (1938); 58, 1 (1939).

[4] E. Wiggin, W. Campbell, O. Maass, Can. J. Research 17, 318 (1939)

[5] J. Fowler and K. Hertel, J. App. Phys. 12, 503 (1941).

[6] R. Sullivan, J. App. Phys. 12, 503 (1941); 13, 725 (1942).

[7] O. Emersleben, Physik. Z. 26, 601 (1925).

[8] R. Tolman, Statistical mechanics with applications to physics and chemistry (Chemical Catalogue Co., New York, N. Y., 1927).

Washington, April 6, 1950.

\title{
Density, Refractive Index, Boiling Point, and Vapor Pressure of Eight Monoolefin (1-Alkene), Six Pentadiene, and Two Cyclomonoolefin Hydrocarbons ${ }^{1}$
}

\author{
By Alphonse F. Forziati, ${ }^{2}$ David L. Camin,${ }^{3}$ and Frederick D. Rossini ${ }^{3}$
}

\begin{abstract}
Density (at $20^{\circ}, 25^{\circ}$, and $30^{\circ} \mathrm{C}$ ), refractive index (at seven wavelengths at $20^{\circ}, 25^{\circ}$, and $30^{\circ} \mathrm{C}$ ), vapor pressure, and boiling point (from 48 to $778 \mathrm{~mm} \mathrm{Hg}$ ) of 16 highly purified samples of hydrocarbons of the API-NBS series were measured for 8 monoolefin (1-alkene), 6 pentadiene, and 2 cyclomonoolefin hydrocarbons.

The data on refractive index were adjusted by means of modified Cauchy and Hartmann equations, and values of the constants are given for each compound.

The data on vapor pressure were adjusted by means of the method of least squares and the three-constant Antoine equation. The values of the constants are given for each compound.

Values were calculated for the specfic dispersions, $\left(n_{F}-n_{C}\right) / d$ and $\left(n_{g}-n_{D}\right) / d$.
\end{abstract}

As a cooperative investigation of the National Bureau of Standards, the U. S. Office of Rubber Reserve, and the American Petroleum Institute Research Project 6, measurements of density, refractive index, vapor pressure, and boiling point were made on highly purified samples of eight monoolefin (1-alkene), six pentadiene, and two cyclomonoolefin hydrocarbons of the API-NBS series.

The compounds measured were made available

This investigation was performed at the National Bureau of Standards as part of the work of the American Petroleum Institute Research Project 6 on the "Analysis, purification, and properties of hydrocarbons."

"Analysis, purification, and properties of hydrocarbons." Project 6.

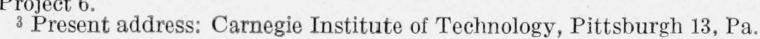

through the American Petroleum Institute Research Project 44 on the "Collection, calculation, and compilation of data on the properties of hydrocarbons." The samples were purified by the American Petroleum Institute Research Project 6 on the "Analysis, purification, and properties of hydrocarbons," from material supplied by the following laboratories:

1-Pentene, by the Phillips Petroleum Co., Bartlesville, Okla.

1-Hexene, 1-heptene, 1-nonene, 1-undecene, and 1,4-pentadiene, by the American Petroleum Institute Research Project 45, at the Ohio State University, Columbus, Ohio. 